\title{
The Perceptions for Hong Kong Male Students Refusing HPV Vaccination: An Exploratory Study
}

\author{
Keung Sum Chan, Lai Hang Wong \\ School of Nursing, Tung Wah College, Hong Kong, China \\ Email:kschan@twc.edu.hk
}

How to cite this paper: Chan, K.S. and Wong, L.H. (2019) The Perceptions for Hong Kong Male Students Refusing HPV Vaccination: An Exploratory Study. Journal of Biosciences and Medicines, 7, 71-84. https://doi.org/10.4236/jbm.2019.75011

Received: April 11, 2019

Accepted: May 14, 2019

Published: May 17, 2019

\begin{abstract}
Background: Human papillomavirus (HPV) is a group of common viruses that can cause a sexually transmitted infection (STI). People with HPV infection can be susceptible to cervical cancer, anogenital warts, and another HPV-related disease. Although the HPV infection can be prevented by HPV vaccination, the vaccination rate in Hong Kong is relatively low particular in males comparing to other countries. Study design: An exploratory qualitative approach was adopted. Aim: This study attempted to explore the perceptions of male college students refusing HPV vaccination in Hong Kong. Methods: A total of 20 male college students participated in the unstructured interview with audio-tape recording and field-notes done to explore their perceptions refusing HPV vaccination. Content analysis was utilized to extract significant themes and sub-themes. Results: Six major themes were identified including a lack of perceived risk, knowledge deficit, inadequate promotion, a high price of the HPV vaccine, inconvenience to receive the HPV vaccine and peer influence. Conclusion: Exploring the perceptions for Hong Kong male college students refusing HPV vaccination could provide essential information to the local government to establish corresponding health campaign for males to increase receiving HPV vaccination for curbing the HPV-related diseases.
\end{abstract}

\section{Keywords}

Perception, Human Papillomavirus Vaccine, Male College Student, Chinese, Qualitative Research

\section{Introduction}

Human papillomavirus (HPV) is a group of common viruses causing sexually transmitted infection (STI) [1]. An HPV infection is well-recognized as a causa- 
tive agent leading to cervical cancer, anogenital warts, and another HPV-related disease. More than 270,000 deaths from cervical cancer are estimated worldwide every year [2]. In the United States, there were $42.5 \%$ adults aged 18 - 59 years have genital infection with any HPV type during 2013-2014 and 66\% of adults having cervical cancers with HPV type 16 and 18 [3]. Besides the United States, cervical cancer has been the seventh commonest cancer among females in Hong Kong [4]. 500 new cases of cervical cancer were diagnosed in 2015 and the incidence rate of having cervical cancer was 12.7 per 100,000 female population [5].

Both men and women have a chance of having HPV infection through sexual contact such as vaginal, anal or oral sex with persons having HPV [3] [6]. Most HPV infections do not cause symptoms or diseases and clear on their own. Nonetheless, genital warts and respiratory papillomatosis can be caused by non-cancer causing types of HPV such as types 6 and 11 [2]. Moreover, HPV can cause cancers including cancers of vulva, vagina, penis or anus [3]. People persistently infected with types 16 and 18 HPV may develop cervical cancer [2].

Despite HPV infection posing a huge impact on health, there are precautionary measures to curb or lower chances of getting HPV infections, which are the cervical screening test and HPV vaccination. The cervical screening test is to detect abnormal cells in the cervix at an early stage [7] and HPV vaccines protect against HPV-related cancers or diseases [3]. According to Centers for Disease Control and Prevention (2017), all 11 to 12-year-old boys and girls start to receive the HPV vaccines and females and males can get the catch-up vaccine until the age of 26 and 21, respectively. Recently, there are 3 registered HPV vaccines protecting from the age of 9 years which are 2-valent vaccine only for female (HPV type 16 and 18), 4-valent vaccine suitable for both sex (HPV type 6, 11, 16 and 18) and 9-valent vaccine for both sex (HPV type 6, 11, 16, 18, 31, 33, 45, 52 and 58) in Hong Kong [8]. The price of the HPV vaccine is around HK\$1500 1700 per dose currently, excluding the medical staffs' fee [9]. There were $24.4 \%$ females under 18 getting HPV vaccine [10]. Although there are safe and effective HPV vaccines, the HPV vaccination rate in Hong Kong is lower than that in other countries.

\section{Literature Review}

A number of studies have investigated attitude, acceptability, and knowledge of HPV vaccination among university students. Hong Kong females deemed that it was critical to be vaccinated against HPV-caused disease before having sex [11]. Compared with the United States, the HPV vaccination rate among female was relatively low in Hong Kong. Several lines of evidence have shown that the cost of HPV vaccines and effectiveness of vaccine could be the major barriers to get HPV vaccinated among female adolescent [11] [12] [13] [14]. Apart from the cost, for parents, the barrier of getting HPV vaccinated included fear of side effects of HPV vaccination and concerning about encouraging sexual activity [15]. Some females were afraid of being label sexually active [10]. 
Furthermore, in a study conducted by Chiang et al. [10], it showed that the HPV vaccination rate of the male was significantly lower than that of the female in Hong Kong. There were many studies on the barrier of HPV vaccinations among males in foreign countries. One of the main reason for men refusing HPV vaccination is they did not perceive HPV as a problem [16] [17]. One of the studies investigated the barriers of getting HPV vaccination among boys in England included a lack of awareness of HPV, lower perceived risk, and fear of injection [18]. However, there is no study on investigating the perception of $\mathrm{HPV}$ vaccination especially targeting college male students in Hong Kong.

\section{Research Gaps and Aim of the Study}

Many studies have investigated the attitude, acceptability, and knowledge of HPV vaccination among university students and adolescents worldwide. Although considerable research has been carried out on studying the barriers of getting HPV vaccinations among males in the foreign countries such as England, and the United States, research focusing on the perceptions of refusing HPV vaccination among male college students in Hong Kong is limited. Therefore, the aim of this study attempted to explore the perception of refusing HPV vaccination among male college students in the local context. The study addressed the following research question: What are the perceptions of Hong Kong male college students refusing HPV vaccination?

Regarding the significance of the study, it provided new insights into identifying the reasons causing male college students to refuse HPV vaccinations so as to increase HPV vaccinations rate and reduce the incidence of HPV-related diseases or cancers among males in Hong Kong.

\section{Study Design}

To explore the research question, a qualitative descriptive approach was employed to address HPV vaccination issues among Chinese male college students.

\section{Setting and Recruitment}

This study was conducted in a selected College in Hong Kong. Convenience sampling with snowball sampling was used to recruit 20 participants for this study in January 2018. The participants were given a \$25 Starbucks voucher as an incentive to participate in the interview. The inclusion criteria for the study participants were (1) Chinese male college students; (2) Age from 18 - 30 years old; (3) Able to speak Cantonese and read Chinese. The exclusion criteria were (1) male college students receiving any dose of HPV vaccines. They were excluded because they had already received the HPV vaccine which would violate the project title. The recruitment of participants was discontinued when the data were saturated.

\section{Instrument}

The interview guide was developed based on the aim of this study, which con- 
sisted of an open-ended question for the unstructured interview. The interview question was included: "Could you tell me why you do not receive HPV vaccines?" The demographic data sheet was adopted to obtain the background information of the participants including age, year in school, academic unit, actual sexual orientation, and sexual history. The field note sheet was used during the interview in order to write down content and participants' emotion as well as other important details was included. A pilot study was conducted to two college male students in order to test the feasibility of the interview guide and the appropriateness of the data collection method. After the pilot study, the interview guide was modified and established to a final version. The skills for taking the field-notes were reviewed in order to facilitate the actual interviews.

\section{Data Collection Method}

Face to face interview was conducted in a discussion room in the library of the selected college from January to March 2018. The room provided a comfortable, quiet environment and privacy for the participants. The unstructured interview was adopted individually by using an interview guide. All interviews were used in Cantonese. All interviews were audio-taped. In order to collect comprehensive information from the individual participants, probing, recapitulation and classification were adopted through the interview. Two researchers were in a group during the interview. One of the researchers asked questions to the participants and the other researcher observed the participants' emotion and took field notes. Each interview was last for approximately 30 to 45 minutes.

Before the interview, the participants did the demographic data sheet. After that, the participants were invited to express the reasons for refusing HPV vaccines. The interviewer encouraged to elaborate more opinions and never urged his opinion to a participant's answer. The observer observed participants' body language and facial expression. During the interview, the interviewer and observer took field notes for recording summary and participants' nonverbal communication.

\section{Data Analysis}

\subsection{Transcribing Interviews}

All audiotaped interviews were transcribed verbatim. Cantonese is a native language for both the researcher and the participants so the researchers were able to transcribe all the interview. To ensure consistency, the researchers took field-note to respective participants for verification of the accuracy and also checked the transcription against the auto-tape interviews. A translator translated transcripts from the original Chinese into English independently. An independent translator being able to speak and read Chinese and English languages fluently translated the English version back into Chinese in order to ensure the consistency. The researcher, who did not involve the interview, compared the Chinese back-translated version and the original Chinese version to identify 
inconsistency. The modified English version was considered as an accurate translation of the original Chinese version.

\subsection{Conducting the Content Analyses}

Qualitative content analysis techniques were adopted to organize and analyze the transcribed data [19]. First, in order to gain a comprehensive sense of the participants' significant ideas, transcripts were read at least three times. Then, all the text was categorized as meaningful units. Next, the categorized meaning of units was abstracted and labeled with different codes. After that, the different codes were compared based on their contrasts and similarities and classified categories and finally, the categories were developed as themes. After developing themes and categories, the researchers then took the findings back and asked the participants for validation so as to check the accuracy.

\section{Trustworthiness}

\subsection{Credibility}

Credibility refers to ensure internal validity [20]. Member checking was adopted in order to ensure credibility. Researchers took the findings back and asked the participants for validation so as to check the accuracy.

\subsection{Dependability}

Dependability refers that a similar finding can be presented in the same approach, the same participants and the same context [20]. A researcher, who did not involve during the interview, compared the Chinese back-translation version and the original Chinese version transcriptions to rectify any errors and omissions in order to evaluate the consistency of the transcriptions.

\subsection{Transferability}

Transferability refers to the degree of this study can be transferred to the other setting [21]. This study provided the characteristics of participants so this study could be as a reference for future study which investigated the perceptions of refusing HPV vaccines among male college students.

\subsection{Confirmability}

Confirmability refers to the findings without any bias. Auto-tape and field notes were contributed to ensuring the accurate meaning of participants. Researcher, who did not involve the interview, compared the Chinese back-translated version and the original Chinese version.

\section{Ethical Consideration}

This study was reviewed and approved by the College Ethics Committee. Before the interview, the participants were informed about the purpose and contents of the study. The information sheet was given and consent form was asked to sign 
to record their voluntary participation. They were informed they had a right to withdraw from the study at any time without giving a reason. The confidentiality of participants' information was assured. All data were accessed only by the researchers involved in the study. Data were stored in an encrypted hard disk. The hard copies and soft copies of the data including the audio recording were put into a locked cabinet. All hard copies and soft copies of the data will be destroyed five years' completion of the study.

\section{Results of the Qualitative Study}

\subsection{Description of the Participants}

The demographic characteristics of the participants are summarized in Table 1. Among 20 participants, 18 of them are heterosexual while 2 of them are homosexual. Half $(n=10)$ of them have a sexual experience. None of them have the history of the sexually transmitted disease. Keeping the anonymity, 20 participants were assigned a code from Mr. A to Mr. T respectively in the study.

From the individual unstructured in-depth interviews of Hong Kong male college students refusing HPV vaccination, six major themes were identified, which were 1) a lack of perceived risk; 2) knowledge deficit; 3) inadequate promotion; 4) a high price of HPV vaccine; 5) inconvenience to receive the HPV vaccine; and 6) peer influence. The themes and categories are shown in Table 2.

Table 1. Demographic Data of the Participants

\begin{tabular}{lcc}
\hline \multirow{2}{*}{ Characteristics } & Demographic characteristics of the participants \\
\cline { 2 - 3 } Study Program & Persons & $(\%)$ \\
Nursing & 8 & 40 \\
Medical Laboratory Science & 5 & 25 \\
Medical Science & 2 & 10 \\
Occupational Therapy & 2 & 10 \\
Applied Gerontology & 1 & 5 \\
Early Childhood Education & 1 & 5 \\
Psychology & 1 & 5 \\
Religion & & \\
No Religion & 13 & 65 \\
Christian & 7 & 35 \\
History of Sexually Transmitted Disease & & 100 \\
No & 20 & 0 \\
Yes & 0 & 50 \\
Sexual Orientation & & 90 \\
Heterosexual & 10 & 10 \\
Homosexual & 2 & \\
Sexual Experience & & 50 \\
Yes & 10 & \\
No & & \\
\hline
\end{tabular}


Table 2. Themes and Categories Generated from the Study

\begin{tabular}{cll}
\hline & \multicolumn{2}{l}{ Themes and Categories } \\
\hline Themes & & \multicolumn{1}{c}{ Categories } \\
\hline A Lack of Perceived risk & - & Considering self as healthy and not getting the illness \\
Knowledge deficit & - & Considering self as a low-risk group \\
& & Inadequate knowledge about the benefits of the HPV \\
Inadequate promotion & - Inadequate HPV vaccine promotion targeting to male \\
A high price of HPV vaccine & - Inade & Unwillingness to pay for HPV vaccine \\
Inconvenience to receive & - Hard to find \\
HPV vaccine & - Time-consuming \\
Peer influence & - Not mentioned from the peer
\end{tabular}

\subsection{A Lack of Perceived Risk}

This theme was generated from the following categories: considering self as healthy and not getting the illness, considering self as a low-risk group.

\subsubsection{Considering Self as Healthy and Not Getting the Illness}

Some participants expressed that they were not in contact with HPV because they deemed themselves as a healthy person and did not have a chance to cause them to illness due to using enough preventive measures during sexual intercourse. These were illustrated by some participants who commented:

"There is no motivation for me to get vaccination... I consider myself having a healthy body, there are no risks that will lead me to illness." (Mr. A: 02)

"When you have preventive methods, like using the condom, it isolated (virus). Therefore, it actually would not have... the bacteria, viruses. That's why I refuse to be vaccinated." (Mr. T: 02)

\subsubsection{Considering Self as a Low-Risk Group}

Some participants believed that HPV was an illness of sexual promiscuity. They expressed that they did not have a high chance to get HPV infection because they did not have multiple sexual partners and have a trustful relationship. These were reported by some participants who commented:

“Since I consider that I won't have the chance, and also I won't involve sexual promiscuity. So, there is no such need. It is not of the high chance for me to get infected with this virus." (Mr. P: 16)

"If it (our relationship) is stable and I trust her, I think there is no such a need. If she is not involving in multiple relationships, her chance of acquiring (HPV) is low, and also for myself. I am not the type of person flirting with others, so I seldom encounter (HPV)...Yup... It is no chance for happening, there is no need for this vaccination when my partner and I wouldn't do that." (Mr. K: 10) 


\subsection{Knowledge Deficit}

The second theme was generated from the category in terms of inadequate knowledge about the benefits of the HPV vaccination to males.

Inadequate Knowledge about the Benefits of the HPV Vaccination to Males

The male misunderstood the purpose of the HPV vaccine. The participants thought that HPV vaccine was effective for females because it was effective in preventing cervical cancer. However, participants reported that they did not know the significance of getting HPV vaccination for males because they did not have the cervix. These were illustrated by some participants who commented:

"I don't know about the benefits? I only know that 'V' stands for the virus, and it has nothing to do with HIV, then it shouldn't be HIV. It is HPV. I thought it is the vaccine for the cervix, so I have not thought of getting vaccinated since I don't have a cervix. However, I know that males can also get vaccinated, but I don't know about the benefits after getting the vaccination. Of course, vaccination is used to prevent illnesses, but I don't really know what exact kind of illness it can prevent." (Mr. A: 09)

"Actually, I think that this is quite effective in preventing cervical cancer in girls, but for boys, I still haven't known... Still, do not think how significant it is. Like, this vaccine... then... yup... (Mr. I: 27); Yup. As I don't know what the effectivenesses are. (Mr. I: 31); Yes... why do I have to get it when I don't know the purpose of it." (Mr. I: 32)

\subsection{Inadequate Promotion}

The third theme emerged from the following categories: inadequate HPV vaccine promotion targeting to male and all population in Hong Kong.

\subsubsection{Inadequate HPV Vaccine Promotion Targeting to Male}

Some participants described promotion mainly focused on cervical cancer induced by HPV in girls while it was less concerning the males and illness caused by HPV for males. This was illustrated by one participant saying:

"People usually promote that this HPV is risky for girls in causing cervical cancer. The focus is mainly on girls. For males, it is not mentioned about what illnesses would be possible (induced by HPV). Without searching for more information, this would increase the chance for males overlooking the impacts of HPV to them... the promotion about its effects and types is insufficient... it (refer to the advertisement) is not mentioned about types of HPV and illness that induced from the HPV can be prevented." (Mr. Q: 25)

\subsubsection{Inadequate HPV Vaccine Promotion Targeting to the Whole} Population in Hong Kong

Some participants mentioned that limited means of HPV vaccine promotion were difficult to approach to all age groups. They believed that teenagers did not 
always watch television, so it was difficult to obtain information about the HPV vaccine through an online platform. One participant shared:

"People in our age range is more active on the internet, they would spend less time on computers ... not computers ... but watching television. So the majority ... I would often watch television when I was small, I know that they have an advertisement. However, for surfing the internet, actually there is not much-related information, or the information pop-ups are not much. Even if you know about it, you have to search for it voluntarily, then you would see it. It is rarely seen that there is a commercial asking you to get HPV vaccinated. So this is less accessible in this aspect." (Mr. O: 07)

\subsection{A High Price of HPV Vaccine}

This theme was generated from the unwillingness to pay for the HPV vaccine.

\section{Unwillingness to Pay for HPV Vaccine}

Most participants mentioned that they would not get HPV vaccinated voluntarily because they deemed that the HPV vaccine was expensive and consider entertainment to be a priority over HPV vaccination. In addition, participants believed that other safety measures were a lower price than HPV vaccine and the acceptable price of vaccine should be within HKD $\$ 1500$. These were reported by some participants who commented:

"If it is to be vaccinated with all 3 shots, I think that the whole course should cost within $\$ 1500$ or within $\$ 1700$. I think that would be better because there would be no one willing to be vaccinated if it costs too much. Plus, just like me, as a student, we don't have much money to do such a thing. We would rather spend our money on playing computer games than to be vaccinated." (Mr. H: 10)

"The price of 1 box of the condom is far cheaper than a single (dose of) vaccine. Since there are other methods (refer to HPV preventive methods) for me to choose, I needn't receive the HPV vaccination." (Mr. Q: 06)

\subsection{Inconvenience to Receive HPV Vaccine}

This theme was generated from the following categories: hard to find and time-consuming.

\subsubsection{Hard to Find}

Most participants understood that it was not available in public hospitals. However, as there was no access to know the exact general practitioner clinics providing HPV vaccination, some participants thought that searching this kind of information was time-consuming and inconvenient. Therefore, this caused them to refuse vaccination. These were reported by some participants who commented:

"I know that it is available in some private clinics, but I don't know exactly which ... clinic is available ... I guess it is like they should be available in those 
private clinics, are they available in public hospitals? Public hospitals don't ... have those, right? If it was available there, then everyone would have been vaccinated in public hospitals. Then, it wouldn't be so inconvenient." (Mr. H: 11)

\subsubsection{Time-Consuming}

Most participants mentioned that they had a busy schedule on schoolwork. As 3-dose HPV vaccination schedule should be administered in several months, some participants deemed that time reserved for different shots of HPV vaccination was a waste of time and they were doubtful of completion of the whole course. There were illustrated by some participants who commented:

"Due to the problem of time constraint ... So yep, I am not vaccinated. (Mr. G: 05); I have to reserve my time, especially for the vaccination if it is separated into different shots... I think it is a waste of time. Also, I may need to wait for several months for vaccinating the next dose. I may not confident enough that I may forget to go for the next vaccination. Then ... therefore, I just let it go, not doing it (the HPV vaccination)." (Mr.G: 14)

"I am a student, there is a lot of school work to do. I have lectures in the morning and afternoon. There are also some private matters at night, after that, I would go to sleep. So, there is not much time for me to think ... this issue." (Mr. O: 06)

\subsection{Peer Influence}

This theme identified from not mentioned from the peer.

\section{Not Mentioned from the Peer}

Friends played a significant role in making the decision because most participants reported that they would follow their friends' actions and suggestions. However, since they believed that society was still traditional, they would not talk about sex-related topic or illness in their social circle. Also, some participants were afraid of having a bad impression if they got HPV vaccinated. This was illustrated by some participants' saying:

“The society is still comparatively traditional. I won't ... won't talk about that with my friends every time, won't talk about having sexual experience, rarely relate to this topic, like the illness, or some problems in this aspect." (Mr. M: 19)

"Um ... The people around me, like if none of your friends are vaccinated, then you would not ... First ... they would not mention it regardless of being vaccinated or not. So, you won't know about it. Second, there is someone who would not dare to get vaccinated if none of his friends are. They would tend to get vaccinated with together with friends." (Mr. O: 22)

\section{Discussion}

This study provided essential information by investigating the perception of Hong Kong male college students refusing HPV vaccination. One of the key findings was the inadequate promotion in Hong Kong related to the importance 
of HPV vaccination for men. A previous study noted that participants expressed a lack of promotion and education about the importance of HPV vaccination for men, so they were not aware of HPV-related diseases affecting both genders [22]. In this study, participants reported that advertisements about HPV vaccination focused on female and preventing cervical cancer while these rarely introduced the significance of HPV vaccination for men.

Participants also described that the theme of advertisements emphasized both genders can get vaccinated rather than telling the importance of HPV vaccination for men or illnesses prevented by the HPV vaccine. Unprecedentedly, this study showed that current means of HPV promotion was limited in Hong Kong. Participants expressed that nowadays advertisements about HPV vaccination mostly promoted through television rather than the Internet and thus the teenagers may not know this kind of information because they would surf the Net to search information as their culture. Therefore, the current promotion of HPV vaccination cannot take all population into consideration.

In addition, male college students may not have adequate knowledge about HPV vaccination. Participants in this study described that the HPV vaccine was effective for the female in preventing cervical cancer. Participants did not think that males had a need to get vaccinated because they did not have the cervix. A similar study conducted in the United States reported that male college students believed the HPV vaccine was not for men and felt confused about whether men could get a vaccine against HPV infection [23]. The current findings concurred with the previous study that HPV infection was a female problem only because much information promoted was targeted at females only, with a clear focus on cervical cancer [24]. This study also showed that male college students did not seem to comprehend the benefits of HPV vaccination towards males. They revealed that they did not know the significance of getting HPV vaccination so they did not think the HPV vaccine was related to them.

Consistent with previous research, the result of this study revealed that male college students believed that the perceived risk of acquiring HPV was low because they consider themselves as a healthy and low-risk group. They deemed that HPV infection was an illness of sexual promiscuity, so they and their partners did not have multiple sexual partners and use safety measures to prevent HPV infection. The previous reports noted that male college students in the United States believed that they used the condom, girlfriends had been virgins and they trusted their partners [23]. Given these reasons, they thought they were not necessary to get vaccinated since they were not at risk of contracting HPV [23].

In this study, male college students did not mention HPV vaccination from the peer. Participants may think that discussing sex-related topics or diseases such as HPV infection was shameful, so they did not talk about these topics or obtain this information such as HPV infection or HPV vaccine in their social circle. People who were more openness to discuss sex-related topics would have a higher intention to get HPV vaccination [25]. This supports that attitude in 
talking about sex-related topics affects the intention of vaccination. On the other hand, this study also showed that male college students being afraid of getting vaccinated against HPV would give other bad impressions. A previous study reported that these participants were worried that they would be labeled sexually active after getting HPV vaccinated [25] [26].

\section{Conclusion}

The findings from this study can help healthcare professionals and researchers understand Hong Kong male college students' perception of refusing HPV vaccination. It showed that promotion about the importance of HPV vaccination for males was not enough. More concerning promotion campaigns on males should be issued in order to increase the awareness of the HPV vaccination among males.

\section{Acknowledgements}

We gratefully acknowledge the participants who have participated in this study. Without their participation, the study would not have been completed. We would like to thank our research team members' great contribution to the research study including Hiu Lam CHAN, Cheuk Chee LI, Mo Kwan WAN, Hong Yiu WU, Yunn Yu CHAN, and Yuen Yee Ho.

\section{Conflicts of Interest}

The authors declare no conflicts of interest regarding the publication of this paper.

\section{References}

[1] Centers for Disease Control and Prevention (2016) What Is HPV? https://www.cdc.gov/hpv/parents/whatishpv.html

[2] World Health Organization (2016) Human Papillomavirus (HPV) and Cervical Cancer. http://www.who.int/mediacentre/factsheets/fs380/en/

[3] Centers for Disease Control and Prevention (2017) Genital HPV Infection-Fact Sheet. https://www.cdc.gov/std/hpv/STDFact-HPV.htm

[4] Department of Health (2017) Statistics. http://www.cervicalscreening.gov.hk/english/sr/sr_statistics.html

[5] Centre for Health Protection (2018) Cervical Cancer. https://www.chp.gov.hk/en/healthtopics/content/25/56.html

[6] Department of Health (2018) What Are Human Papillomavirus (HPV) and the HPV Vaccine? https://www.fhs.gov.hk/english/health_info/faq/women_health/WH2_5_2.html

[7] The Chinese University of Hong Kong (2016) What Is Cervical Cancer? http://www.cuhk.edu.hk/sphpc/hpvselfsampling/en/cc-n-hpv.html

[8] Department of Health (2016) Human Papillomavirus (HPV) Vaccine. https://www.cervicalscreening.gov.hk/english/hum/hum_ccv.html

[9] Hong Kong Cancer Fund. (n.d.) Cervical Cancer Vaccine. 
http://www.cancer-fund.org/cervical/eng/vaccine/vaccine.html

[10] Chiang, V.C.L., et al. (2016) Attitude, Acceptability and Knowledge of HPV Vaccination among Local University Students in Hong Kong. International Journal of Environmental Research and Public Health, 13. https://doi.org/10.3390/ijerph13050486

[11] Kwan, T.T.C., et al. (2008) Barriers and Facilitators to Human Papillomavirus Vaccination among Chinese Adolescent Girls in Hong Kong: A Qualitative-Quantitative Study. Sexually Transmitted Infections, 84, 227-232. https://doi.org/10.1136/sti.2007.029363

[12] Li, S.L., et al. (2013) HPV Vaccination in Hong Kong: Uptake and Reasons for Non-Vaccination amongst Chinese Adolescent Girls. Vaccine, 31, 5785-5788. https://doi.org/10.1016/j.vaccine.2013.10.027

[13] Choi, H.C., Leung, G.M., Woo, P.P., Jit, M. and Wu, J.T. (2013) Acceptability and Uptake of Female Adolescent HPV Vaccination in Hong Kong: A Survey of Mothers and Adolescents. Vaccine, 32, 78-84. https://doi.org/10.1016/j.vaccine.2013.10.068

[14] Chan, K.K.L., et al. (2012) Human Papillomavirus Vaccine: What Are Women Most Concerned about? Journal of Obstetrics and Gynaecology Research, 38, 23-30. https://doi.org/10.1111/j.1447-0756.2011.01639.x

[15] Radisic, G., Chapman, J., Flight, I. and Wilson, C. (2017) Factors Associated with Parents' Attitudes to the HPV Vaccination of Their Adolescent Sons: A Systematic Review. Preventive Medicine, 95, 26-37. https://doi.org/10.1016/j.ypmed.2016.11.019

[16] Liddon, N., Hood, J., Wynne, B. and Markowit, L. (2010) Acceptability of Human Papilla Marvirus Vaccine for Males: A Review of the Literature. Journal of Adolescent Health, 46, 113-123. https://doi.org/10.1016/j.jadohealth.2009.11.199

[17] Crosby, R., DiClemente, R., Salazar, L., Nash, R. and Younge, S. (2011) Gardasil for Guys: Correlates of Intent to Be Vaccinated. Journal of Men Health, 8, 119-125. https://doi.org/10.1016/j.jomh.2011.03.001

[18] Forster, A.S., Marlow, L.A.V., Wardle, J., Stephenson, J. and Waller, J. (2012) Interest in Having HPV Vaccination among Adolescent Boys in England. Vaccine, 30, 4505-4510. https://doi.org/10.1016/j.vaccine.2012.04.066

[19] Graneheim, U.H. and Lundman, B. (2004) Qualitative Content Analysis in Nursing Research: Concepts, Procedures and Measures to Achieve Trustworthiness. Nurse Education Today, 24, 105-112. https://doi.org/10.1016/j.nedt.2003.10.001

[20] Shenton, A.K. (2004) Strategies for Ensuring Trustworthiness in Qualitative Research Projects. Education for Information, 22, 63-75. https://doi.org/10.3233/EFI-2004-22201

[21] Curtin, M. and Fossey, E. (2007) Appraising the Trustworthiness of Qualitative Studies: Guidelines for Occupational Therapists. Australian Occupational Therapy Journal, 54, 88-94. https://doi.org/10.1111/j.1440-1630.2007.00661.x

[22] Fontenot, H.B., Fantasia, H.C., Vetters, R. and Zimet, G.D. (2016) Increasing HPV Vaccination and Eliminating Barriers: Recommendations from Young Men Who Have Sex with Men. Vaccine, 34, 6209-6216. https://doi.org/10.1016/j.vaccine.2016.10.075

[23] Fontenot, H.B., Collins Fantasia, H., Charyk, A. and Sutherland, M.A. (2014) Human Papillomavirus (HPV) Risk Factors, Vaccination Patterns, and Vaccine Perceptions among a Sample of Male College Students. Journal of American College Health, 62, 186-192. https://doi.org/10.1080/07448481.2013.872649

[24] Prue, G., Shapiro, G., Maybin, R., Santin, O. and Lawler, M. (2016) Knowledge and 
Acceptance of Human Papillomavirus (HPV) and HPV Vaccination in Adolescent Boys Worldwide: A Systematic Review. Journal of Cancer Policy, 10, 1-15.

https://doi.org/10.1016/j.jcpo.2016.09.009

[25] Di Giuseppe, G., Abbate, R., Liguori, G., Albano, L. and Angelillo, I.F. (2008) Human Papillomavirus and Vaccination: Knowledge, Attitudes, and Behavioural Intention in Adolescents and Young Women in Italy. British Journal of Cancer, 99, 225-229. https://doi.org/10.1038/sj.bjc.6604454

[26] Wong, L.P. (2008) Young Multiethnic Women's Attitudes toward the HPV Vaccine and HPV Vaccination. International Federation of Gynecology and Obstetrics, 103, 131-135. https://doi.org/10.1016/j.ijgo.2008.07.005 